\title{
Stochastic block models with multiple continuous attributes
}

\author{
Natalie Stanley ${ }^{1,2^{*}}$, Thomas Bonacci ${ }^{3}$, Roland Kwitt ${ }^{4}$, Marc Niethammer ${ }^{5}$ and Peter J. Mucha ${ }^{6}$
}

\author{
${ }^{*}$ Correspondence: \\ stanleyn@stanford.edu \\ ${ }^{1}$ Curriculum in Bioinformatics and \\ Computational Biology, University \\ of North Carolina at Chapel Hill, \\ Chapel hill, USA \\ ${ }^{2}$ Present address: Stanford \\ University, Stanford, USA \\ Full list of author information is \\ available at the end of the article
}

\begin{abstract}
The stochastic block model (SBM) is a probabilistic model for community structure in networks. Typically, only the adjacency matrix is used to perform SBM parameter inference. In this paper, we consider circumstances in which nodes have an associated vector of continuous attributes that are also used to learn the node-to-community assignments and corresponding SBM parameters. Our model assumes that the attributes associated with the nodes in a network's community can be described by a common multivariate Gaussian model. In this augmented, attributed SBM, the objective is to simultaneously learn the SBM connectivity probabilities with the multivariate Gaussian parameters describing each community. While there are recent examples in the literature that combine connectivity and attribute information to inform community detection, our model is the first augmented stochastic block model to handle multiple continuous attributes. This provides the flexibility in biological data to, for example, augment connectivity information with continuous measurements from multiple experimental modalities. Because the lack of labeled network data often makes community detection results difficult to validate, we highlight the usefulness of our model for two network prediction tasks: link prediction and collaborative filtering. As a result of fitting this attributed stochastic block model, one can predict the attribute vector or connectivity patterns for a new node in the event of the complementary source of information (connectivity or attributes, respectively). We also highlight two biological examples where the attributed stochastic block model provides satisfactory performance in the link prediction and collaborative filtering tasks.
\end{abstract}

Keywords: Stochastic block model, Networks, Community detection, Attributes

\section{Introduction}

Uncovering patterns in network data is a common pursuit across a range of fields, such as in biology (Larremore et al. 2013), medicine (Aghaeepour et al. 2017; Guinney et al. 2015) and computational social science (Greene and Cunningham 2013). Community detection is a powerful algorithmic way to understand the large-scale structural organization of a network (Porter et al. 2009; Leskovec et al. 2010; Shai et al. 2017; Fortunato 2010; Fortunato and Hric 2016). In this pursuit, the objective is to identify cohesive groups of nodes with relatively high density of within-group connections and fewer between-group connections. Numerous approaches exist to accomplish this task, but typically only the adjacency matrix encoding connectivity patterns is taken into account. In various applications, each node in a network is equipped with additional information (or particular

(c) The Author(s). 2019 Open Access This article is distributed under the terms of the Creative Commons Attribution 4.0 International License (http://creativecommons.org/licenses/by/4.0/), which permits unrestricted use, distribution, and reproduction in any medium, provided you give appropriate credit to the original author(s) and the source, provide a link to the Creative Commons license, and indicate if changes were made. 
attributes) that was not implicitly taken into account in the construction of the network. For example, in a protein interaction network, each protein could contain multiple experimental measurements or classifications.

Significant attention has been given to the interplay between connectivity-based (or structural) community organization of the network and the attribute information of nodes within communities (Bothorel et al. 2015). Importantly, it is often unclear whether it is valid to assume that a structural community should necessarily correlate with an attribute-based functional community (Hric et al. 2016; Peel et al. 2017; Yang and Leskovec 2015). While such studies suggest that extreme caution should be taken in assuming a correlation between structural and functional communities, we limit our focus in the present work to the assumption that a node's connectivity and attribute patterns can be jointly modeled based on its community assignment. In other words, we seek to develop an approach to assign nodes to communities based jointly on both sources of information, such that a community is defined as a group of nodes with similar connectivity and attribute patterns. In doing so, our objectives are three-fold: first, we develop a probabilistic approach to jointly model connectivity and attributes; second, we wish to ensure that our model can handle multiple, continuous attributes; third, we demonstrate the utility of the fitted model for link prediction and collaborative filtering applications.

The rest of this paper is organized as follows. This section includes subsections on related work in attributed networks and stochastic block models, as background. We then describe our model and associated inference procedure in "Model" section. In "Synthetic data results" section, we demonstrate the behavior of the model fitting synthetic data. We then apply the model for the tasks of link prediction and collaborative filtering in "Using the fitted attributed SBM for link prediction and collaborative filtering" section, followed by applications in biological networks examples in "Applications in biological networks" section.

\section{Related work in attributed networks}

Recently, there have been numerous efforts to incorporate attribute information into the community detection problem (Yang et al. 2013; Newman and Clauset 2016; Combe et al. 2015; Hric et al. 2016; Peel et al. 2017). In describing our contribution, we distinguish between methods that descriptively obtain communities through optimization of a quality function and those that generatively capture communities through probabilistic models. Quality function based methods define a quantity of interest that an ideal partition would satisfy, while probabilistic methods identify communities through likelihood optimization and focus on the underlying statistical distribution for the observed network. A recent quality function-based method to handle multiple attributes is I-louvain (Combe et al. 2015). This method approaches the problem as an extension to the Louvain algorithm, which is the state-of-the-art scalable modularity quality function community detection method (Blondel et al. 2008). The modularity-based approach to community detection defines a null model for community structure under the assumption that there is not substantial structural organization in the network and seeks to identify a partition maximally different from this model through optimizing the modularity quality function. The I-louvain method modifies the standard modularity quality function to what they label 'inertia-based modularity', incorporating a Euclidean distance between nodes based on their attributes, and demonstrating with multiple examples how incorporating 
connectivity and attributes allows for a partition of nodes to communities that aligns better with ground truth than that obtained using connectivity or attributes in isolation.

Alternatively, there are a variety of probabilistic approaches for handling attributed network data (Newman and Clauset 2016; Hric et al. 2016; Peel et al. 2017; Yang et al. 2013). Similar to our work in the sense that community membership is related to node attributes is CESNA (Yang et al. 2013). The objective in this approach is to learn a set of propensities or affiliations for each node across all possible communities, such that two nodes with similar propensities towards communities should have more in common in terms of connectivity and attributes. In this model, each node has a vector with multiple binary attributes. The affiliation model is useful and flexible because it does not enforce a hard partitioning of nodes into communities, which is useful in social network applications. In this inference problem, the connectivity and attribute information are used to infer a node's affiliations to communities and then models the probability of an edge between two nodes as a function of the similarity in their community affiliation propensities.

In contrast to the affiliation model, the stochastic block model (Snijders and Nowicki 1997) (at least the more standard variants of it), seeks to determine a hard partition of nodes across communities and models edges between a pair of nodes according to their community assignments. The partition of nodes to communities through a stochastic block model framework is accomplished through maximum likelihood optimization. A variant of the stochastic block model explored by Newman and Clauset (Newman and Clauset 2016) adapts the classic stochastic block model to handle a single (possibly continuous) attribute with the assumption that attributes (referred to as 'metadata') and communities are correlated. Importantly, the Newman and Clauset approach does not assume any particular form for the corresponding mixture model, expressing the model as a series expansion in Bernstein polynomials whose coefficients are inferred as part of the algorithm. In contrast, in order to most simply simultaneously include multiple continuous attributes in our model, we make particular assumptions about the underlying mixture model, demonstrating the utility of our approach by way of the applications considered below. As the geometry of attribute data in high dimensions can be highly variable across datasets, having more than one attributed stochastic block model is advantageous. For example, in a dataset with multiple attributes one could apply principal component analysis (PCA) to the data to determine how many principal components are needed to capture a suitable amount of variance in the data. If only a single component is needed, then the Newman and Clauset approach is a very suitable choice. If more principal components are needed to capture sufficient variation in the data, our approach can be very useful. Further work is needed to define how to best use the geometry of the attribute space to apply the most appropriate version of the attributed SBM, but this is beyond the scope of this paper.

Hric et al. (2016) developed an attributed SBM from a multilayer network perspective, with one layer modeling relational information between attributes and the other modeling connectivity, then assigning nodes to communities maximizing the likelihood of the observed data in each layer. Finally, work by Peel et al. (2017) made important contributions in 1) establishing a statistical test to determine whether attributes actually correlate with community structure and 2) developing an SBM with flexibility in how strongly to couple attributes and community membership in the stochastic block model inference problem. In contrast to the SBM extension proposed by Newman and Clauset, where 
the attributes are used to build a prior for node-to-community assignments (Newman and Clauset 2016), the SBM adaptation by Peel et al. learns whether a node's community assignment should be chosen through inference or should be fixed as its metadata label.

The model that we seek to develop in this work is distinguished by its ability to fit a stochastic block model to undirected networks where each node has multiple continuous attributes. We assume that attributes are some complementary source of information from the data used to specify connectivity between nodes. This model is most appropriate for situations where a node's community membership determines its connectivity (e.g. traditional SBM) and attribute information. While the model proposed by Newman et al. can accommodate continuous attributes (Newman and Clauset 2016), we view our contribution as a method to consider multiple continuous attributes simultaneously and to learn a generative model that describe the multidimensional attribute vectors across communities. We model the multidimensional attribute vectors for each nonoverlapping community by a particular multivariate Gaussian model, which allows us to sample attributes from the model (in the case of missing attributes) or to understand the consensus mean and covariance for members of a community. We highlight two applications of our attributed SBM in "Applications in biological networks" section, to a protein interaction network and a microbiome subject similarity network. Before discussing these examples, we first define our attributed SBM and an inference technique for fitting the model. We test this approach on a synthetic example. Since community detection methods are often difficult to validate due to the lack of ground truth information on the nodes, we describe the tasks of link prediction and collaborative filtering to quantify how well the attributed SBM represents the data. We then consider these tasks on two biological network examples.

\section{Stochastic block models}

Because our model is an extension to the widely-used stochastic block model (Snijders and Nowicki 1997), we provide a brief introduction here. This model assumes that edges within a community are connected within and between communities in a characteristic or probabilistic way. To fit this model to network data, the objective is to partition the nodes into communities such that these assignments maximize the likelihood of the model according to the observed edges. In this inference problem for a network with $N$ nodes and $K$ communities, one learns a $K \times K$ probability matrix, $\boldsymbol{\theta}$, describes the probability of connections within and between communities, and an $N$-length vector of node-to-community assignments, $\mathbf{z}$. For a network with $N$ nodes, $K$ communities, adjacency matrix, $\mathbf{A}=\left\{a_{i j}\right\}$ and a learned vector $\mathbf{z}$ of node-to-community assignments, the SBM without degree correction (degree-corrected versions also exist Karrer and Newman (2011)) models an edge between nodes $i$ and $j$ with

$$
a_{i j} \sim \operatorname{Bernoulli}\left(\boldsymbol{\theta}_{z_{i} z_{j}}\right)
$$

The node-to-community assignments $(\mathbf{z})$ are inferred together with the matrix $\theta$ through likelihood optimization. Effective inference techniques for standard stochastic block model parameters are well explored (Zhang et al. 2012; Peixoto 2014; Daudin et al. 2008), including algorithms for EM, belief propagation, and MCMC accept-reject sampling. 


\section{Model}

\section{Objective}

We seek to incorporate both connectivity $(\mathbf{A})$ and attribute information $(\mathbf{X})$ to infer nodeto-community assignments, $\mathbf{Z}$. Note that for a network with $N$ nodes, $K$ communities and $p$ measured attributes, $\mathbf{A}=\left\{a_{i j}\right\}, \mathbf{X}=\left\{x_{i p}\right\}$, and $\mathbf{Z}=\left\{Z_{i k}\right\}$ have dimensions $N \times N, N \times p$ and $N \times K$, respectively. In particular, we distinguish $\mathbf{Z}$ to be a binary indicator matrix, where entry $Z_{i c}$ is 1 if and only if node $i$ belongs to community $c$, whereas we also define $\mathbf{z}$ to be the equivalent $N$-dimensional array labeling node-to-community assignments. We assume connectivity and attributes are conditionally independent, given the community membership label. The graphical model for the relationship between node-to-community labels, connectivity and attribute information is shown in Fig. 1.

To infer the $\mathbf{Z}$ that best explains the data, we adopt a likelihood maximization approach. That is, we seek to find the partition of nodes to communities that best describes the observed connectivity and attribute information. Given the conditional independence assumption of $\mathbf{X}$ and $\mathbf{A}$, we can express the log likelihood of the data, $\mathcal{L}$ as the sum of connectivity and attribute log likelihoods, $\mathcal{L}_{A}$ and $\mathcal{L}_{X}$, respectively, as

$$
\mathcal{L}=\mathcal{L}_{A}+\mathcal{L}_{X}
$$

This likelihood reflects the joint distribution of the adjacency matrix, $\mathbf{A}$, the attribute matrix, $\mathbf{X}$, and the matrix of node-to-community indicators, $\mathbf{Z}$; formally, we have

$$
\mathcal{L}=p(\mathbf{A}, \mathbf{X}, \mathbf{Z}) .
$$

Given that $\mathbf{Z}$ is a latent variable that we are trying to infer, we can approach the problem using the expectation maximization (EM) algorithm (Dempster et al. 1977). By doing this, we will alternate between estimating the posterior probability that a node $i$ has community label $c$, or

$$
p\left(Z_{i c}=1 \mid \mathbf{X}, \mathbf{A}\right)
$$

and estimates for $\boldsymbol{\theta}, \boldsymbol{\Psi}$, which specify the adjacency matrix and attribute matrices, respectively.

\section{Attribute likelihood}

For a network with $K$ communities, we assume that each particular community $i$ has an associated $p$-dimensional mean $\boldsymbol{\mu}_{i}$ and $p \times p$ covariance matrix, $\boldsymbol{\Sigma}_{i}$. Note

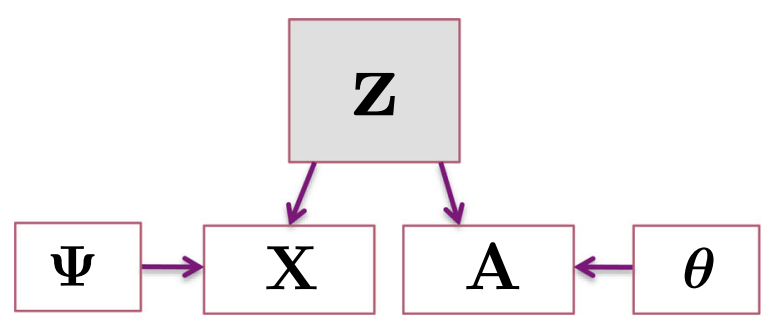

Fig. 1 Modeling community membership in terms of attributes and connectivity. Node-to-community assignments specified by $\mathbf{Z}$ are determined in terms of adjacency matrix information, $\mathbf{A}$ and attribute matrix information, $\mathbf{X}$. A and $\mathbf{X}$ are assumed by be generated from a stochastic block model and a mixture of multivariate Gaussian distributions, parameterized by $\boldsymbol{\theta}$ and $\boldsymbol{\Psi}$, respectively 
that these parameters uniquely identify a $p$-dimensional multivariate Gaussian distribution. To specify this model for all $K$ communities, we define the parameter $\boldsymbol{\Psi}=$ $\left\{\mu_{1}, \mu_{2}, \ldots \mu_{k}, \Sigma_{1}, \Sigma_{2}, \ldots \Sigma_{K}\right\}$.

The log likelihood for the mixture of Gaussians on the attributes is written as,

$$
\log (p(\mathbf{X} \mid \boldsymbol{\Psi}))=\sum_{i=1}^{N} \log \left\{\sum_{c=1}^{K} \pi_{c} \mathcal{N}\left(\mathbf{x}_{i} \mid \boldsymbol{\mu}_{c}, \mathbf{\Sigma}_{c}\right)\right\}
$$

Here, $\mathcal{N}\left(\mathbf{x}_{i} \mid \boldsymbol{\mu}_{c}, \boldsymbol{\Sigma}_{c}\right)$ is the probability density function for the multivariate Gaussian and $\pi_{c}$ is the probability that a node is assigned to community $c$.

\section{Adjacency matrix likelihood}

For the adjacency matrix, $\mathbf{A}$ and the $K \times K$ matrix of stochastic block model parameters, $\boldsymbol{\theta}$, Daudin et al. showed (Daudin et al. 2008) that the complete data log likelihood can be expressed as

$$
\begin{aligned}
\log (P(\mathbf{A} \mid \mathbf{Z})) & =\frac{1}{2} \sum_{i \neq j} \sum_{k, l} Z_{i k} Z_{j l}\left[a_{i j} \log \left(\theta_{k l}\right)\right. \\
& \left.+\left(1-a_{i j}\right) \log \left(1-\theta_{k l}\right)\right] .
\end{aligned}
$$

\section{Inference}

To use EM (Dempster et al. 1977) to maximize the likelihood of the data, we break the process into the E-step and M-Step, and perform this step sequence iteratively until the estimates converge.

E-Step. During the E-step, we use the current value of learned model parameters, $\boldsymbol{\theta}$ and $\boldsymbol{\Psi}$ to compute the posterior given in Eq. (4) at each step. The posterior at each step, $\gamma\left(Z_{i c}\right)$, of node $i$ belonging to community $c$, is given by

$$
\begin{aligned}
\gamma\left(Z_{i c}\right) & =p\left(Z_{i c}=1 \mid \mathbf{x}_{i}, \mathbf{a}_{i}\right) \\
& =\frac{p\left(\mathbf{x}_{i} \mid Z_{i c}=1\right) p\left(\mathbf{a}_{i} \mid Z_{i c}=1\right) \pi_{c}}{\sum_{k=1}^{K} p\left(\mathbf{x}_{i} \mid Z_{i k}=1\right) p\left(\mathbf{a}_{i} \mid Z_{i k}=1\right) \pi_{k}} .
\end{aligned}
$$

Here, $\mathbf{x}_{i}$ and $\mathbf{a}_{i}$ denote the attribute and connectivity patterns for node $i$, respectively. In particular, $\mathbf{a}_{i}$ is the $i$ th row of the adjacency matrix, A. Therefore, the $j$ th entry of $\mathbf{a}_{i}$ is 1 if nodes $i$ and $j$ are connected and 0 otherwise.

M-Step. In the M-step, we can compute updates for $\boldsymbol{\theta}$ and $\boldsymbol{\Psi}$ using this expectation.

Since the attributes follow a Gaussian mixture model, the update for the mean vector describing community $c, \boldsymbol{\mu}_{c}$, can be computed using the EM algorithm (Dempster et al. 1977) as

$$
\boldsymbol{\mu}_{c}=\frac{\sum_{i=1}^{N} \gamma\left(Z_{i c}\right) \mathbf{x}_{i}}{\sum_{i=1}^{N} \gamma\left(Z_{i c}\right)}
$$

Similarly, the update for the covariance matrix describing a community, $\boldsymbol{\Sigma}_{c}$, is computed as

$$
\boldsymbol{\Sigma}_{c}=\frac{\sum_{i=1}^{N} \gamma\left(Z_{i c}\right)\left(\mathbf{x}_{i}-\boldsymbol{\mu}_{c}\right)\left(\mathbf{x}_{i}-\boldsymbol{\mu}_{c}\right)^{T}}{\sum_{i=1}^{N} \gamma\left(Z_{i c}\right)}
$$


To update the parameters of $\boldsymbol{\theta}$, we follow the method in Daudin et al. (2008) and update the probability of an edge existing between community $q$ and $l$, given by $\boldsymbol{\theta}_{q l}$ as,

$$
\boldsymbol{\theta}_{q l}=\frac{\sum_{i \neq j} \gamma\left(Z_{i q}\right) \gamma\left(Z_{j l}\right) a_{i j}}{\sum_{i \neq j} \gamma\left(Z_{i q}\right) \gamma\left(Z_{j l}\right)}
$$

The updates shown in Eqs. (8) and (9) and standard mixture of Gaussians updates (Bilmes and et al 1998). The update in Eq. (10) was shown by Daudin et al. (2008).

We continue the process of iterating between the E-step and M-step until the change in the data $\log$-likelihood, $\mathcal{L}$, is below a predefined tolerance threshold. Once the loglikelihood has converged, the value for each $Z_{i c}$ can be interpreted as follows. The $Z_{i c}$ corresponding to node $i$ is 1 if $c$ is the community corresponding to the largest posterior probability $\left(\gamma\left(z_{i c}\right)\right.$ across all $c$. Moreover $Z_{i c}=0$ for all communities $c$, such that $c \operatorname{did}$ not correspond to the highest posterior probability. Similarly, $\sum_{c} Z_{i c}=1$.

\section{Initialization}

Likelihood optimization approaches are often sensitive to initialization because it is easy to get stuck in a local optimum. As an initialization strategy for the nodes, we simply cluster the nodes in the network using the Louvain algorithm (Blondel et al. 2008).

\section{Synthetic data results}

We first test the performance of our model and inference procedure on a synthetic example. We generated networks with a stochastic block model with $N=200$ nodes and $K=4$ communities, parameterized as follows:

$$
A_{i j} \sim \begin{cases}\operatorname{Bernoulli}(.10), & \text { if } z_{i} \neq z_{j} \\ \operatorname{Bernoulli}(.25), & \text { if } z_{i}=z_{j}\end{cases}
$$

Note that $\mathbf{z}$ is a 200-dimensional vector, where $z_{i}$ identifies the community label for node $i$.

Figure 2a shows the adjacency matrix for an example network generated according to this parametrization. The black marks in the image indicate edges. While this network has assortative structure with members of a community having more edges on average with each other than with other communities, there are still many noisy edges going between communities, making the correct community structure more difficult to discern.

To model attributes, for a community $c$, we randomly generated an 8-dimensional vector, $\boldsymbol{\mu}_{c}$, where each entry is from a Gaussian with 0 -mean and unit variance. Associated with each $c \in\{1,2,3,4\}$ is an $8 \times 8$ diagonal covariance, $\boldsymbol{\Sigma}_{c}=\operatorname{diag}(1.25)$. Moreover, using the $\boldsymbol{\mu}_{c}$ and $\boldsymbol{\Sigma}_{c}$, a sample attribute vector can be generated. That is, the attribute vector $\mathbf{x}_{i}$ for node $i$ is generated as

$$
\mathbf{x}_{i} \sim \mathcal{N}\left(\boldsymbol{\mu}_{z_{i}}, \boldsymbol{\Sigma}_{z_{i}}\right)
$$

where $\mathcal{N}(\cdot, \cdot)$ denotes a multivariate Gaussian.

Figure $2 \mathrm{~b}$ shows a PCA plot of the attribute vectors associated with each node in an example synthetic experiment, with each point representing a node. Since the true dimension of these feature vectors is eight, this plot provides a projection onto the first two principal components, allowing a visualization of the relatedness between node attributes. One can observe that members of community two are overall nicely separated 


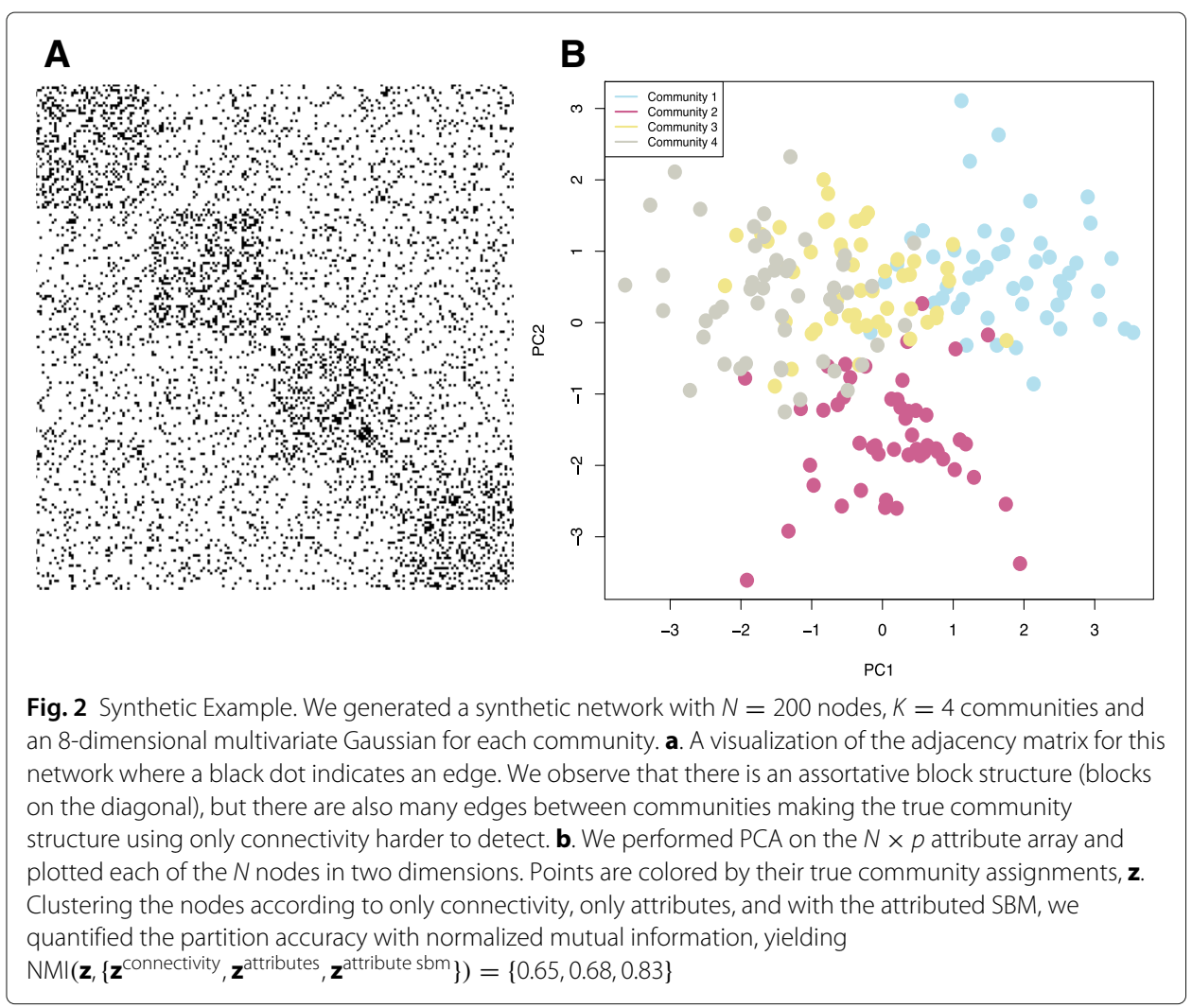

from other communities in the projected attribute space but members of communities three and four are especially hard to discern here.

To assess how well the attribute SBM approach performed in successfully assigning nodes to communities, we compared the results obtained from our model to clustering results obtained clustering based only on connectivity and to clustering based only on the attribute information. We quantify the correctness of the obtained partitions with normalized mutual information (NMI) (Danon et al. 2005). Letting $\mathbf{z}$ denote the true node-to-community assignments, then $\mathbf{z}^{\text {connectivity }}, \mathbf{z}^{\text {attributes }}$, and $\mathbf{z}^{\text {attribute sbm }}$ denote the partition of the nodes according to the network connectivity only, attributes only, and with the attributed SBM. To cluster the network only according to connectivity, we fit a stochastic block model with four blocks. To cluster nodes with only attributes, we performed $k$-means clustering on only the attributes. Computing the NMI between $\mathbf{z}$ and each of these three cases, we obtain $0.65,0.68$, and 0.83 , respectively. These results show that by combining both sources of information, there is an improvement in the ability to correctly identify communities. To further probe this idea, we sought to empirically look closer at the so-called 'detectability limit'. Generally, detectability refers to the difficulty of correctly identifying clusters in data; in particular, sharp phase transitions are observed in fitting stochastic block models, with accurate capture of the correct communities only if the within-community probability, $p_{i n}$, is sufficiently larger than the between-community probability, $p_{\text {out }}$ (Decelle et al. 2011; Taylor et al. 2016).

Based on the results of the synthetic experiments in Fig. 2 where the attributes combined with connectivity lead to a more accurate partitioning of the nodes, we 
hypothesized that augmenting the network connectivity with attributes may move this detectability limit. In Fig. 3, we explored how generating networks from a stochastic block model with varying ratios between $p_{i n}$ and $p_{\text {out }}$ combined with the attributes used in Fig. 2 would affect the accuracy of the node-to-community partition. To do this, we considered values of $p_{\text {in }}$ between 0.05 and 0.3 in increments of 0.05 . For each of these $p_{\text {in }}$ values, we found the corresponding value of $p_{\text {out }}$ such that the mean degree was 20 . Fixing the mean degree allows for direct comparison of how the within-to-between community probabilities influence the detection of correct communities. For each of these $p_{i n}$ and $p_{\text {out }}$ combinations, we generated 10 different networks using a stochastic block model. In Fig. 3 we plot the NMI between the true partition, $\mathbf{z}$ and the partitions using only the connectivity with the regular SBM $\mathbf{z}^{\text {connectivity }}$ and the attributed SBM $\mathbf{z}^{\text {attribute }}$ sbm. These results are plotted in blue and pink, respectively. The shaded region around the points indicates standard deviation.

We see that while both inference approaches undergo a strong increase in accuracy at a similar ratio of $p_{\text {in }} / p_{\text {out }}=3$, we notice that the curve for the attribute SBM results are slightly shifted to the left due to the use of the extra attribute information positively impacting the ability to correctly identify communities. Moreover, we note that the attribute SBM results appear to smooth out the sharp phase transition that is visible in the results from the SBM without attributes. Future work could focus on better understanding the impact on such detectability questions in terms of the parameters for the underlying multivariate Gaussian distributions parametrizing each community.

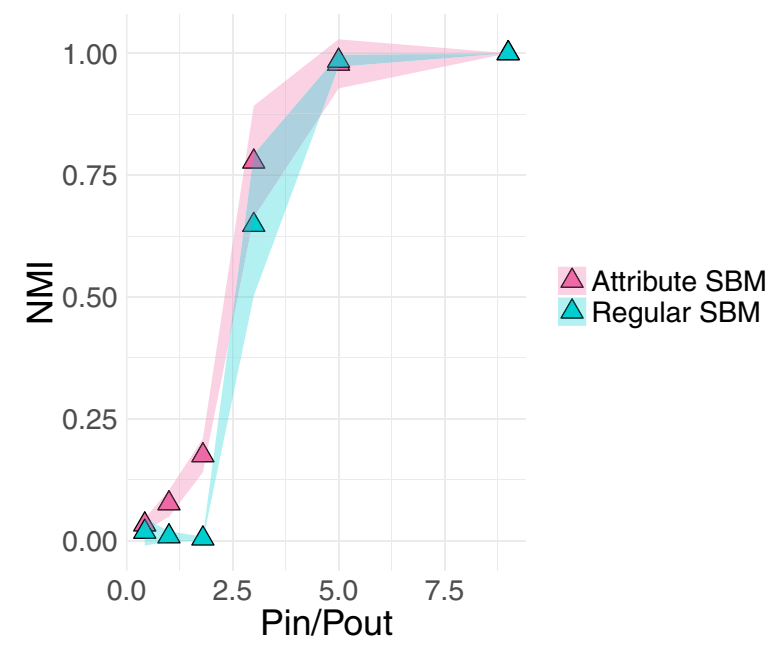

Fig. 3 Detectability Analysis in Synthetic Example. To understand how attribute information can be combined with connectivity to assign nodes to communities accurately, we generated synthetic networks for within-probabilities of $p_{\text {in }}$ between 0.05 and 0.3 with corresponding $p_{\text {out }}$ or between-community probabilities such that the mean degree of the network was 20. For each of these synthetic networks, we used the attributes from the analysis in Fig. 2 to fit the attributed SBM. Here, we plot the correctness of the node-to-community assignment with normalized mutual information using the partition obtained from regular SBM (blue) and the partition under the attributed SBM model fit (pink). For each combination of $p_{\text {in }}$ and $p_{\text {out }}$, we generated 10 networks and hence the bands around the points denote standard deviation. Incorporating attributes with the attributes stochastic block model improves results, particularly near and below the detectability limit, and appears to smooth out the sharp phase transition 


\section{Using the fitted attributed SBM for link prediction and collaborative filtering}

One of the benefits of a generative network model is that it can be applied to prediction tasks. Most notably, in the absence of one source of information about a node (connectivity or attributes), the model can be used to predict the complementary information source (attributes or connectivity, respectively). We demonstrate here that fitting an attributed SBM may provide a means to successfully perform two fundamental network prediction tasks: link prediction and collaborative filtering.

In the link prediction problem, when given two node stubs, the objective is to determine whether a link exists between them. Since we are modeling connectivity with a stochastic block model, we can predict links using the learned parameters. In particular, we highlight how this task can be performed using just the attribute information of the node stubs of interest. In the experiments to follow, we compare to 3 commonly-used link prediction methods. In all of these methods, a score is computed for all edge-candidate dyads and ultimately the top $x$ set of prospective edges with highest weights are kept (where $x$ is some user-defined parameter). Let $i$ and $j$ be a pair of nodes and $\Gamma(i)$ denote the set of neighbors for a node $i$. Then, under the following 3 common link prediction methods (Wang et al. 2014), we can calculate the score of the potential link as Score $(i, j)$.

Jaccard: $\operatorname{Score}(i, j)=\frac{\Gamma(i) \cap \Gamma(j)}{\Gamma(i) \cup \Gamma(j)}$

Adamic Adar: $\operatorname{Score}(i, j)=\sum_{c \in \Gamma(i) \cap \Gamma(j)} \frac{1}{\log |\Gamma(c)|}$

Preferential Attachment: $\operatorname{Score}(i, j)=|\Gamma(i)| \times|\Gamma(j)|$

Stochastic Block Model: We also compare to the plain stochastic block model (SBM) by using a standard stochastic block model to predict edge probabilities according to the learned parameters according to connectivity only. To predict the probability that an edge exists between nodes $i$ and $j$, we fit a plain SBM based only on connectivity. This results in a matrix of between-community probability parameters, $\boldsymbol{\theta}$ and a vector of node-tocommunity assignments $\mathbf{z}$. Then we specify the probability that an edge exists between nodes $i$ and $j$ as $\theta_{z_{i}, z_{j}}$.

Conversely, the collaborative filtering problem seeks to predict a node's attributes based on its similarity to its neighbors. For some node of interest, we can use our fitted attributed SBM model to predict a node's attributes, given only the information about its connectivity. Formally, for node $i$, we seek to predict $\mathbf{x}_{i}$. In the following experiments, we compare our results to two common collaborative filtering approaches (Shi et al. 2014). Let $\mathcal{N}^{k}(i)$ be the set of $k$-nearest neighbors in the network for node $i$. Let $\hat{\mathbf{x}}_{i}$ be the predicted attribute vector for node $i$ and $s_{i j}$ be a similarity measure (e.g. correlation) encoded through the weighted edge between nodes $i$ and $j$.

$$
\begin{aligned}
& \text { Neighborhood Avg: } \hat{\mathbf{x}}_{i}=\frac{1}{\left|\mathcal{N}^{k}(i)\right|} \sum_{j \in \mathcal{N}^{k}(i)} \mathbf{x}_{j} \\
& \text { Weighted Neighborhood Avg: }
\end{aligned}
$$

$$
\hat{\mathbf{x}}_{i}=\frac{1}{\sum_{j \in \mathcal{N}^{k}(i)} s_{i j}} \sum_{j \in \mathcal{N}^{k}(i)} s_{i j} \mathbf{x}_{j}
$$

Stochastic Block Model (SBM): Similar to our link prediction experiments, we also use the plain stochastic block model as another baseline prediction method. To do this in a particular network, we identify its communities and compute an approximation $\left(\hat{\boldsymbol{\mu}}_{c}\right)$ of the mean attribute vector $\left(\boldsymbol{\mu}_{c}\right)$ in community $c$ as simply the mean of the attributes associated with the nodes assigned to community $c$. For a node $i$, we identify its nearest 
neighbors again in connectivity space. Then we predict the attribute vector $\hat{\mathbf{x}}_{i}$ for node $i$ as $\hat{\boldsymbol{\mu}}_{c}$, where $c$ is the most common community among nearest neighbors.

We show results for these two tasks in two different biological network examples in "Applications in biological networks" section. In particular, the experiments were designed in the following ways.

\section{Link prediction experiments}

For the link prediction tasks shown in Figs. 4 and 5, we performed a link prediction task by sampling pairs of nodes and utilizing the complementary source of attribute information. We sampled 10 different sets of 50 pairs of nodes. In each sample, 25 of the node pairs were those having an edge in the original network and 25 were pairs with no edge. For each of the 50 edges in each sample, we sought to predict whether an edge existed between the corresponding node pair in a leave one out manner. To do this, for each edge we fit the attributed SBM to the network with the pair of nodes (stubs) associated with the edge removed. We then use the nearest neighbor in attribute space of each stub as the input to each of the three baseline link prediction methods (Jaccard, Adamic Adar, and Preferential Attachment). To use our attributed SBM in this link prediction task, we also consider the most commonly observed community among the 3 nearest neighbors for the stubs $i$ and $j$ of the edge of interest. We denote the label for the most common community among the three-nearest neighbors of nodes $i$ and $j$ as $z_{i}$ and $z_{j}$, respectively. We then define the link prediction score for the edge as $\theta_{z_{i}, z_{j}}$, or the probability

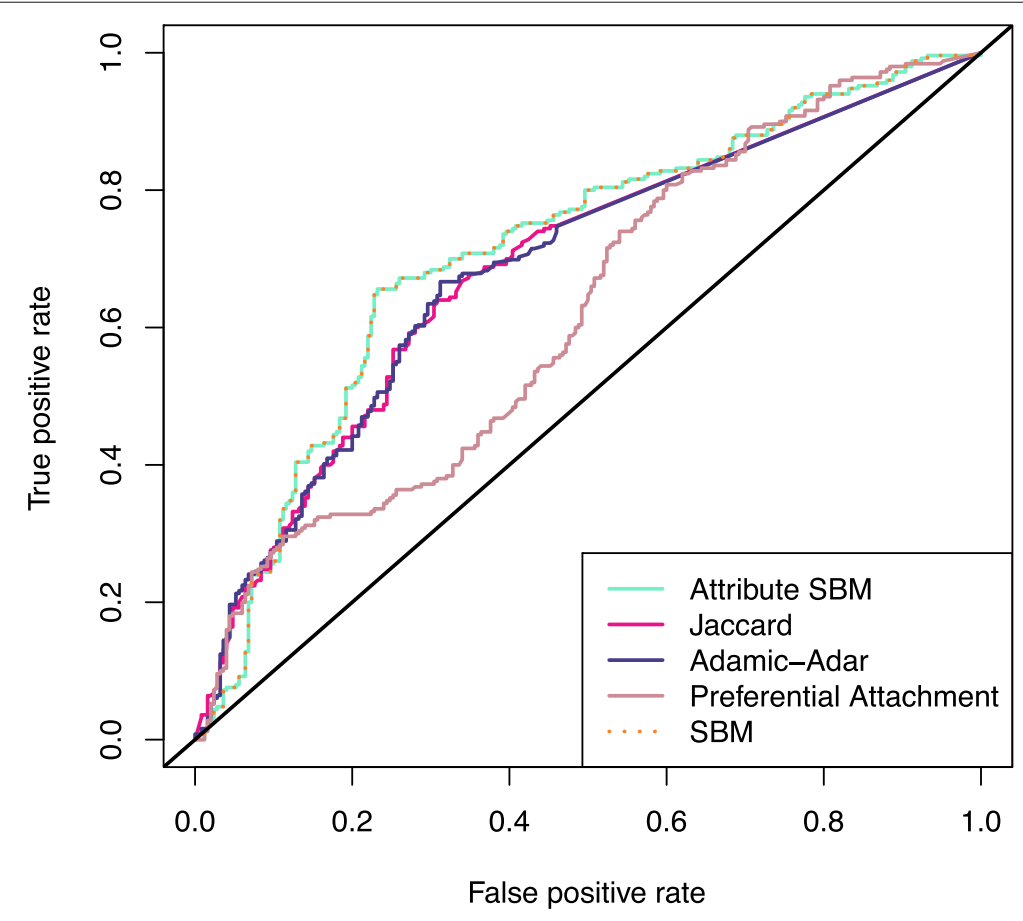

Fig. 4 Link Prediction on the microbiome subject similarity network: The results for link prediction on the microbiome subject similarity network for the attributed SBM, Jaccard, Adamic-Adar, preferential attachment, and SBM methods. The corresponding AUC values for these methods, respectively are, $0.71,0.69,0.69$, and 0.62 , and 0.71 , respectively 


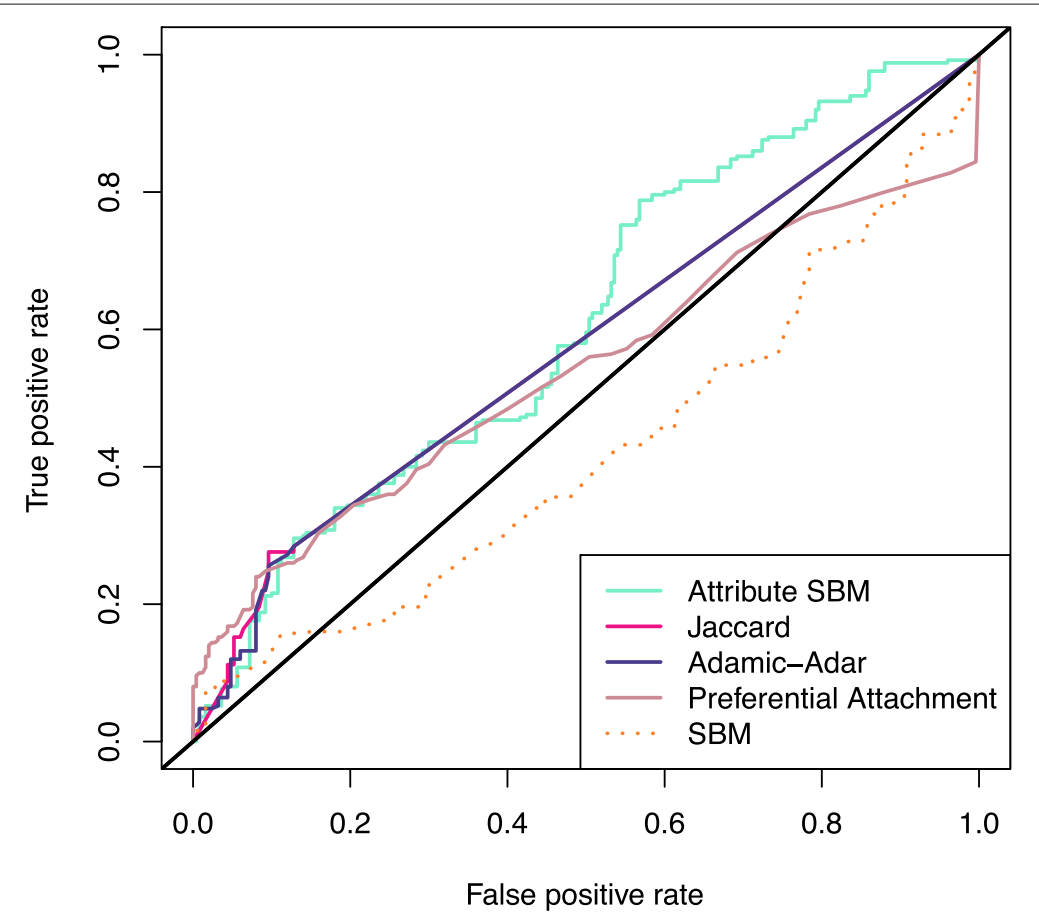

Fig. 5 Link Prediction in the protein interaction network. Performing link prediction using the attributed SBM, Jaccard, Adamic Adar, preferential attachment, and SBM. The corresponding AUC curves for these methods were $0.61,0.58,0.58,0.54$, and 0.42 , respectively

that an edge exists between nodes $i$ and $j$ according to the fitted model. After generating 10 samples of 50 edge pairs, this results in 500 total edge scores. Since we know the ground truth of whether or not these edges actually exist from the original network, we can construct an ROC curve for each method. From these curves we can plot area under the curve (AUC) to quantify the quality of the link prediction result. Using the attributed SBM is a way to incorporate community information into the link prediction problem which has previously shown to be effective (Soundarajan and Hopcroft 2012). In "Applications in biological networks" section we show that the attributed SBM offers the highest performance (area under the ROC curve) on two biological datasets, in comparison to baseline methods.

\section{Collaborative filtering experiments}

In collaborative filtering experiments, the objective is to predict the vector of attributes for each node. In our experiments, we used leave-one-out validation to predict the attribute vector for each node. That is, for each node in the network, we created a single node test set. The training set, was then the rest of the network with the node to predict removed. For this single test set node, we identified neighbors it connects to in only connectivity space within the training set. For standard collaborative filtering approaches (Neighborhood average and weighted neighborhood average), the predicted attribute for the test set node is then the specified averaging of the neighbors. To use our model for this task, we first fit the attributed SBM model to the training set. Similar to the standard link prediction approaches, we identify the nearest neighbors for our test node in connectivity space within the training set. We then predict the community membership of 
our test node to be the most-frequently observed community among its neighbors. Using this community assignment, $c$, we can then predict the attribute vector, $\mathbf{x}_{i}$ for a node $i$ from the fitted attribute SBM model. In particular, the predicted value for $\mathbf{x}_{i}$ is a sample from the learned multivariate Gaussian distribution for community $c$. This model is parameterized by mean, $\boldsymbol{\mu}_{c}$ and covariance $\boldsymbol{\Sigma}_{c}$. These results are shown in Figs. 6 and 7 . For a node $i$ and its associated vector of attributes, $\mathbf{x}_{i}$ we quantify the accuracy of the predicted attribute vector, $\hat{\mathbf{x}}_{i}$ with root mean squared error (RMSE). We denote the error for a particular collaborative filtering approach by $\mathcal{E}$ and for a particular node it quantifies the difference between the true and predicted attribute values $\left(\mathbf{x}_{i}\right.$ and $\left.\hat{\mathbf{x}}_{i}\right)$.

Similar to the success of integrating community information for link prediction, collaborative filtering tasks have previously shown success from the integration of network community structure (Deng et al. 2014). In "Applications in biological networks" section, we show that using the attributed SBM for collaborative filtering tasks leads to the lowest error compared to baselines on two biological datasets.

\section{Applications in biological networks}

We evaluated the potential to combine similarity or relational information between a set of entities for application in biological data. For example, one might consider networks of proteins, genes, or bacterial species with extra experimental data. Our application of this model to biological problems provides a framework to predict attribute or connectivity information about a new observation. Note that we do not intend to suggest any new biological insights here, but rather that we can combine two sources of information for prediction tasks and alternative definitions of what constitutes a community in the data. Applying the attributed stochastic block model to integrate connectivity and attribute data provides a way to find a partition that takes into account two different sources of information, or a method to predict one source of information (connectivity, attributes) in the absence of the other (attributes, connectivity).

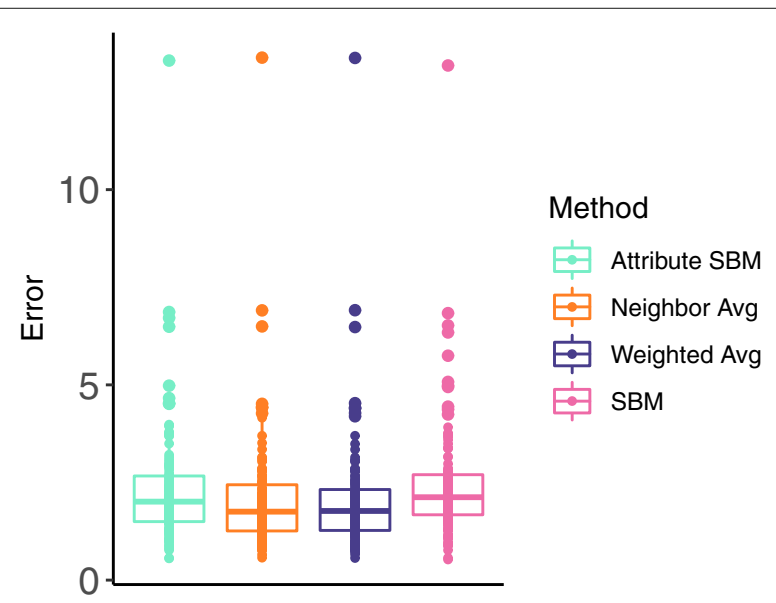

Fig. 6 Collaborative Filtering Accuracy in Microbiome Subject Similarity Network: For each of the 121 nodes, we fit a model to the remaining 120 node network and given the node's closest neighbors (based on network connectivity) sought to predict its 5-dimensional attribute vector. The reported error is the root mean squared error (RMSE) (denoted by $\mathcal{E}$ ) between the true attribute vector $\left(\mathbf{x}_{i}\right)$ and its predicted attribute vector $\left(\hat{\mathbf{x}}_{i}\right)$. The mean root squared error in the attributed SBM is 2.3, as opposed to the neighbor average, weighted neighbor average and SBM having mean errors of 2.06, 2.05, and 2.45, respectively 


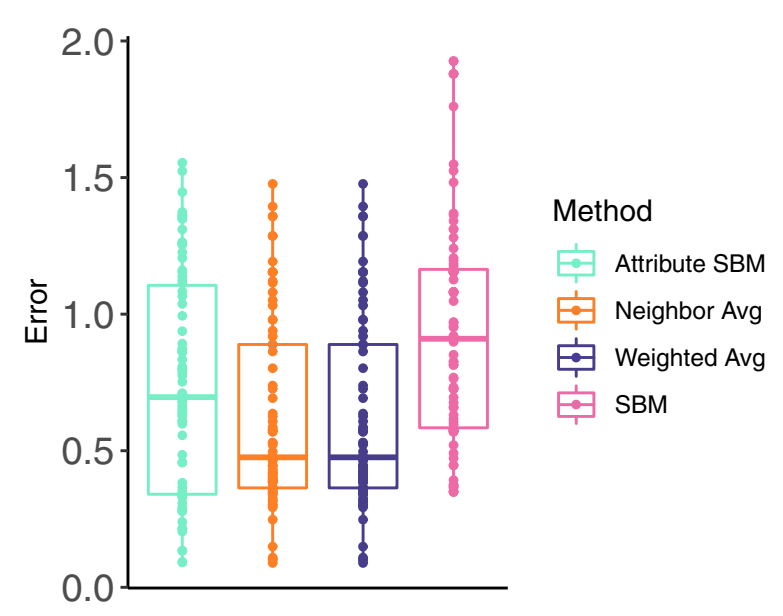

Fig. 7 Collaborative filtering in the protein interaction network. For each of the 82 nodes, we fit a model to the remaining 81 node network and given the node's closest neighbors (based on network connectivity) sought to predict its 6-dimensional attribute vector. The reported error is the root mean squared error (denoted by $\mathcal{E}$ ) between the difference between the true attribute vector $\left(\mathbf{x}_{i}\right)$ and its predicted attribute vector $\left(\hat{\mathbf{x}}_{i}\right)$. The mean error in $\mathbf{x}_{i}$ using the attributed SBM is 0.74 , as opposed to the neighborhood average, weighted neighborhood average, and SBM, resulting in errors of $0.74,0.62,0.62$, and 0.94 , respectively

\section{Microbiome subject similarity results}

\section{Motivation}

In the analysis of biological data, it is often useful to cluster subjects based on a set of their measured biological features and to then determine what makes each of the subgroups different. One type of biological data gaining much attention in recent years is metagenomic sequencing data, used to profile the composition of a microbiome. We refer to this as the 'metagenomic profile' and each feature is a count for each bacterial species, also known as operational taxonomic unit (OTU). Lahti et al. conducted a study among subjects across a variety of ethnicities, body mass (BMI) classifications, and age groups to understand differences in the intestinal microbiota (Lahti et al. 2014). Using metagenomic sequencing, the counts for 130 OTUs were provided for each subject. Using the OTU profiles, we created an attributed network between subjects based on their similarities in OTU profiles. In this case, connectivity represents pairwise correlation between subjects based on their OTU profiles. The attributes for each node are defined as the coordinates for a lower-dimensional embedding (described in the next section). In this case, we are overlaying two similar ways to look at the data and examining how this can enhance results. Our work here is not meant to reveal novel biology, but rather to provide a computational test for understanding the performance of our attributed SBM approach.

Pre-Processing The data were downloaded from http://datadryad.org/resource/doi:10. 5061/dryad.pk75d. We extracted a subset of the subjects from Eastern Europe, Southern Europe, Scandinavia, and the United States. Using only these subjects, a between-subject similarity network was constructed between the 121 individuals who had a BMI measurement. This resulted in a network of 121 nodes, where each edge is the Pearson correlation between their microbial compositions. We then removed all edges in the network with weight (correlation) $<0.7$. Note that our attributed SBM does not allow for edge weights, so we simply ignored the edge weights as input to the model. 
Constructing Node Attributes Since each node had a 130-dimensional vector of attributes (counts), we used this information to create a lower-dimensional attribute vector for each node by performing PCA and then representing each node with the first 5 principal components. Each dimension of this new attribute vector was then centered and scaled, and we observed an approximately Gaussian distribution.

We first visualized the differences in partitions obtained according to the classic and attributed stochastic block models in Fig. 8a-b, respectively. In both networks, nodes are colored by their community assignment. Using the classic stochastic block model and the model selection criterion described in (Daudin et al. 2008), 7 blocks were identified. With the attributed stochastic block model, 6 blocks were identified. While we do not have ground truth labels on the nodes, it is visually apparent that adding the attributes to the inference problem helps to 'clean up' the partition. For example, in Fig. 8a there is mixing between communities two and three in the network colored by communities identified with the regular SBM. In Fig. 8b, this mixing was reduced after using the attributed SBM by assigning all of the nodes in the general region to community three. Similarly, in Fig. 8a, members of community five are periphery nodes that are connected well to community seven but not to each other. Because these nodes do not have a lot of internal connectivity, it is not immediately apparent why they were assigned to be in their own community. This nuance is corrected in Fig. 8b with the use of the attributed stochastic block model. Here, the members of communities five and seven from Fig. 8a. are mostly agglomerated into community six. The layout for each of these networks was computed using the 'layout.nicely' function in the R igraph library.

Microbiome Link Prediction We performed link prediction on the microbiome subject similarity network as described in "Link prediction experiments" section. The associated ROC curves are plotted in Fig. 4. All five methods have satisfactory performance with the attributed stochastic block model giving the best results. The AUC values

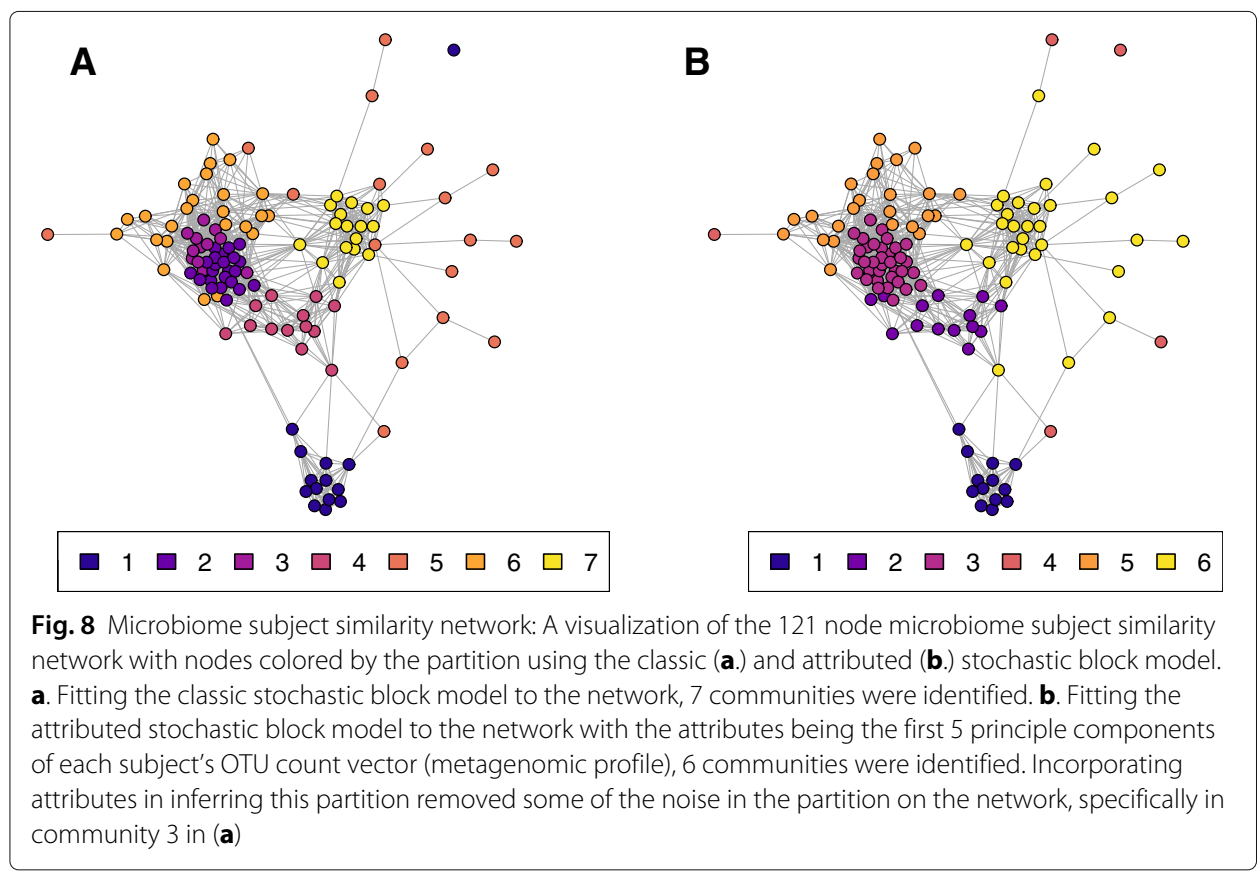


for the attributed SBM, Jaccard, Adamic-Adar, preferential attachment, and SBM are 0.71, $0.69,0.69,0.62$, and 0.71 , respectively.

We note that the performance of the attributed SBM and SBM are very similar in the microbiome network shown in Fig. 4. This likely arises from the fact that the node attributes and connectivity are quite similar. Alternatively in Fig. 5 the attributes and connectivity are more complementary sources of information. Moreover, including the attribute information greatly helps the link prediction task in this case.

Microbiome Collaborative Filtering We performed the collaborative filtering experiments on the microbiome subject similarity network in the manner described in "Collaborative filtering experiments" section to predict the 5-dimensional attribute vector for each node. The box plots in Fig. 6 indicate the distribution of root mean squared error (RMSE), $\mathcal{E}$, over the 121 nodes for the attribute SBM (turquoise), neighbor average (orange), weighted neighbor average (blue), and SBM (pink). We find that in general the edge-based collaborative filtering methods (neighbor average and weighted neighbor average) have better performance than community model-based predictions (attributed SBM, SBM). This is likely because predicting an attribute vector based on an average over a collection of nodes likely introduces more noise than predicting only based on closest neighbors. A similar behavior is also observed in the collaborative filtering experiments in the protein interaction network shown in Fig. 7. In general, all four methods have similar error distributions, but the attributed SBM outperforms the standard SBM. The mean RMSEs for the attributed SBM, neighbor average, weighted neighbor average, and SBM were 2.3, 2.06, 2.05, and 2.45, respectively. This experiment along with the experiment shown in Fig. 7 show that while not quite as accurate as the methods based on closest neighbors, the attributed stochastic block model nevertheless can be effectively used for collaborative filtering tasks. A possibly interesting direction for future work would be to consider combinations of these methods from different perspectives to attempt to identify greater improvements in accuracy.

\section{Protein interaction network results}

We also apply our attributed SBM approach to the protein interaction network presented in (Bonacci et al. 2014). This network represents interactions between proteins, predicted from the literature. Associated with each node (protein), is a classification of one of 6 experimental modifications observed from the exposure of cancer cells to a chemotherapeutic drug. While communities in this network should reflect functional relatedness among proteins (e.g. similar biological functions, in general), we also expect that members of a community should share similarities in the observed modification type. Also associated with each of the 6 modification types is whether that particular type of modification became either more or less prominent after treatment with the drug. Since we have two types of labels associated with these nodes, we also sought to explore how these two labeling schemes ( 6 class vs. 2 class) aligned with the communities returned by the algorithm.

Data Pre-Processing: We downloaded the unweighted protein interaction network data and the modification information from the supplement of (Bonacci et al. 2014). We removed 13 nodes that were not connected to the largest component of the network and considered only the 82 node largest connected component. 
Constructing Node Attributes: Each node is classified with 1 of 6 possible modification types. For each node, we created an attribute vector that captured the modification types of its neighbors. To do this, we considered the 4th order neighborhood of each node. That is, for each node, we collected its neighbors who were four hops or less away in the network. Then to define the value for attribute $c$ of node $i$, or $x_{i c}$, we counted the number of 4th order neighbors of node $i$ with label $c$. After defining these attributes across all nodes, for each of the 6 classes, we centered and scaled each attribute across all of the nodes to have mean 0 and unit variance.

Figure 9a-b show the results of fitting a classic SBM and attributed SBM, respectively. These networks are visualized with the Fruchterman Reingold force directed layout in the R igraph library. Nodes are colored by their community assignment. The 6 possible modifications arise from 3 biological processes that can either increase or decrease after exposure to the drug. The node shape reflects whether the experimental modification for a node increased (square) or decreased (circle) after treatment with the chemotherapeutic agent. Again by fitting an SBM with the model selection criterion in (Daudin et al. 2008), five communities were identified. With our attributed SBM, nine communities were identified. Note that using the attributed SBM created more communities in that it split up the purple core community under the classic SBM into more small communities. The implications of this new partition are explored with an entropy calculation based on the biological classifications of the protein in Fig. 10.

Using the partition of the nodes under the classic and attributed stochastic block models, we sought to use the two different prior biological classifications or labeling of the nodes (proteins) (Bonacci et al. 2014). The first of these classifications includes one of six modification types. The other classification refers to whether the protein has increased or decreased levels of expression. Moreover, we could use these two types of labels or classifications of the proteins to compute a label entropy measure within each community. The expectation is that by incorporating attribute information that is related to the functional

A

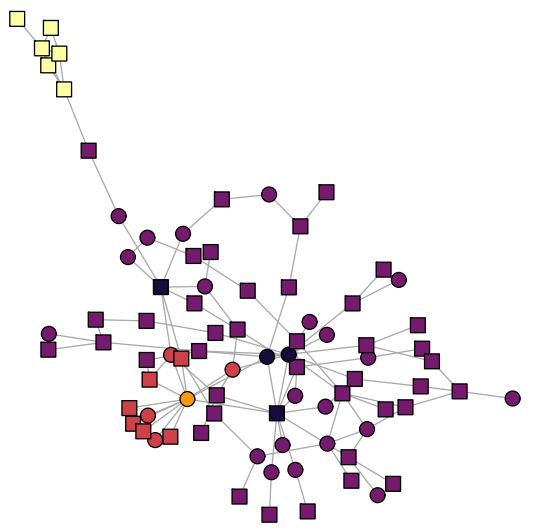

B

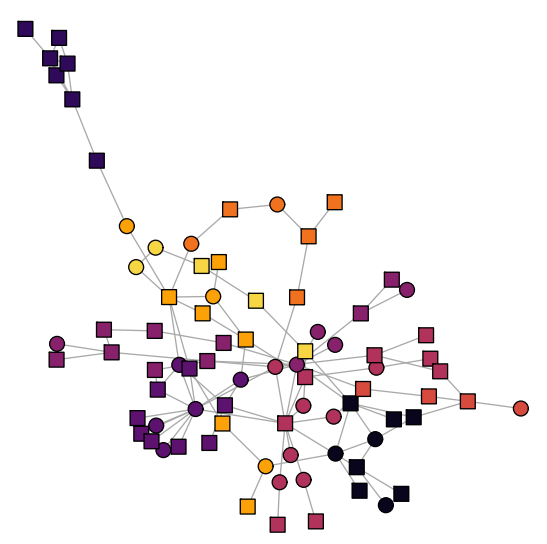

Fig. 9 Protein interaction network. We visualize the 82 node protein interaction network under the classic stochastic block model (a.) and the attributed stochastic block model (b.) In both networks, nodes are colored by their community assignment and the node shape indicates whether the modification status increased (square) or decreased. a. Nodes colored according to the community partition under the stochastic block model. Nodes are assigned to one of five communities. b. Nodes are colored to the community partition under the attributed SBM. Nodes are assigned to one of nine communities 

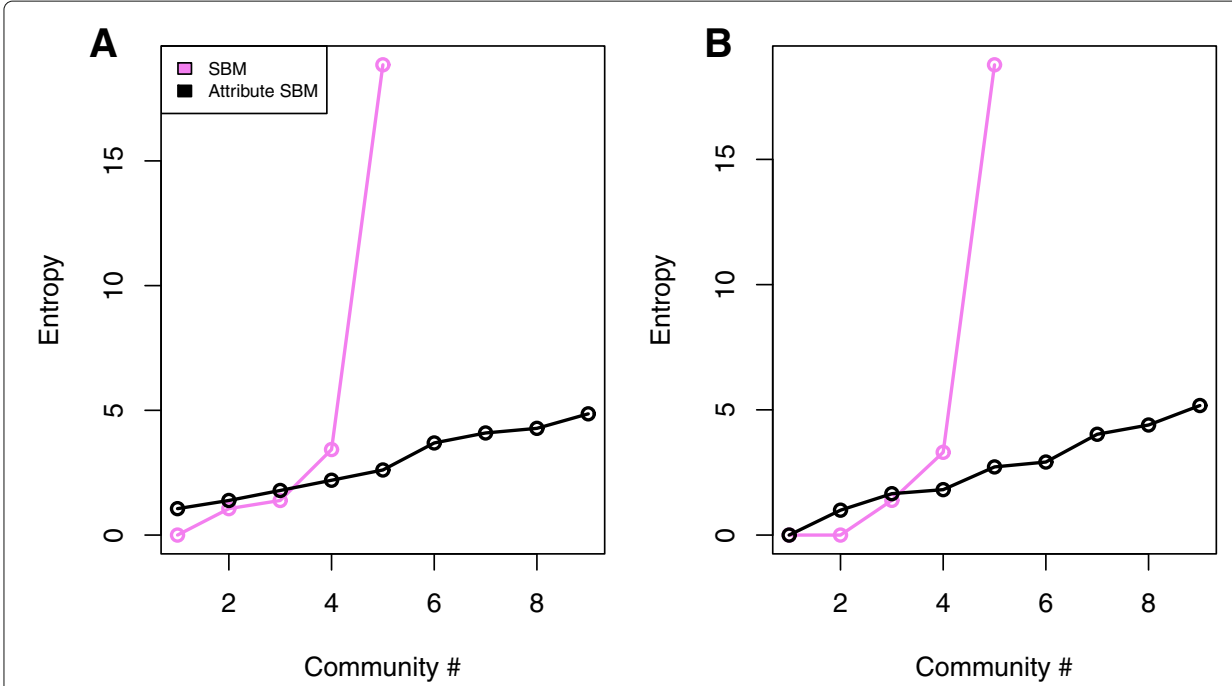

Fig. 10 Community entropies in the protein interaction network. We studied the entropy of the 2 class and 6 class classifications of the nodes in $\mathbf{a}$. and $\mathbf{b}$., respectively under the classic SBM (black) and attributed SBM (purple) partitions. For A - B. the horizontal axis denotes the community index for the particular partition. Nodes belonged to 1 of five communities under the classic SBM and belong to one of nine communities under the attributed SBM. Incorporating attributes under both classifications succeeds in breaking up a high e ntropy community (5) from the classic SBM partition to lower entropy communities in the attributed SBM partition

protein information into the community detection problem, we should see a decrease in the entropy over the biological classification labels in communities. In Fig. 10a-b, we plot the entropy for the 2 class and 6 class node classifications, respectively. We define $\mathbf{E}_{c}$, the entropy for community $c$ as

$$
\mathbf{E}_{c}=-\sum_{k} p_{k} \log \left(p_{k}\right)
$$

Here, $k$ indexes the unique classifications found in community $c$ and $p_{k}$ is the probability that a node in community $c$ belonged to biological classification $k$ in community $c$. In these plots the black and purple curves correspond to the fits of the classic and attribute SBM fits, respectively. Using both types of node classifications to compute these entropy quantities, we see that the attribute SBM succeeds in breaking up one high entropy community (5) from the classic SBM partition into lower entropy communities.

Link Prediction in the Protein interaction network We performed link prediction on the protein interaction network using the procedure described in "Link prediction experiments" section. Given that this protein network is sparse, none of the link prediction methods performed particularly well. The AUC values for the attributed SBM, Jaccard, Adamic-Adar, preferential attachment, and SBM were 0.61, 0.58, 0.58, 0.54, and 0.42, respectively. The associated ROC curves are shown in Fig. 5. In this example, the attributed SBM significantly outperforms the regular SBM. This is most likely due to the complementary and non-overlapping nature of the connectivity and attribute information.

Collaborative filtering in the protein interaction network Collaborative filtering was performed using the method described in "Collaborative filtering experiments" section. Note that unlike the microbiome sample similarity network, the edges in this network 
are unweighted and hence the neighbor average and weighted neighbor average methods produce the same result. Similar to the results in the microbiome network in Fig. 6, we notice that edge-based methods (neighborhood average and weighted neighborhood average) result in a lower error than community-based methods (attributed SBM, SBM). Similarly, the attributed SBM has higher performance over the plain SBM. The root mean squared errors for the attributed SBM, neighborhood average, weighted neighborhood average and SBM were 0.74, 0.62, 0.62, and 0.94, respectively. Clearly, the attributed SBM offers several advantages over the original SBM. Similar to Fig. 6, the box plots in Fig. 7 represent the distribution of errors across each of the 82 nodes.

\section{The attributed SBM leads to increased network assortativity}

We further quantified the quality of the partition obtained by integrating network connectivity and node attributes with an assortativity measure (Newman 2003). Assortativity measures the extent of homophily in a network, under some labeling of the nodes. Intuitively, this implies that in a network with a high assortativity coefficient, nodes tend to have the same labels as their neighbors. Moreover, we labeled nodes according to their community assignments under the classic and attributed stochastic block model and computed assortativity using the assortativity function in igraph. We found that in both the microbiome and protein dataset, using the attributed SBM leads to higher assortativity. In Fig. 11 we show that the assortativities for the regular and attributed SBMs in the microbiome data were 0.61 and 0.71 , respectively. Similarly, in the protein dataset, the assortativity measures for the regular and attributed SBM were -0.122 and 0.714 , respectively. The assortativity computed in the protein-protein interaction network with the

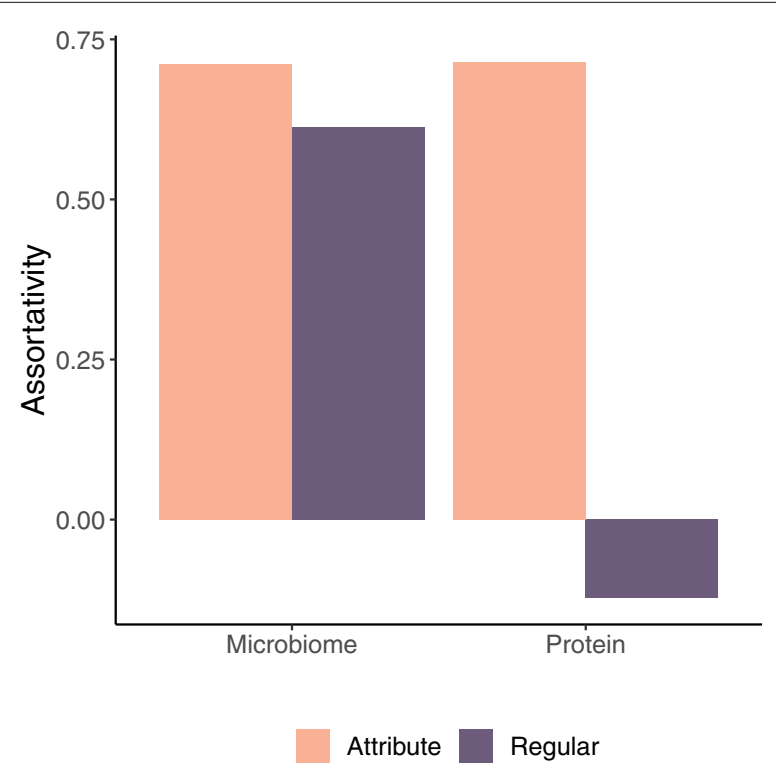

Fig. 11 Assortativity for class and attributed stochastic block models. We computed assortativity coefficients for both the microbiome subject similarity and protein-protein interaction networks. The node-to-community assignments under the regular SBM (purple) and attributed SBM (orange) were used to define node labels when computing assortativity. In both datasets, the use of the attributed SBM lead to higher assortativity measures 
regular SBM was negative. This implies that the induced labels are not coherent between individual nodes and their neighbors.

\section{Conclusions and future work}

We defined an attributed stochastic block model, where a node's community assignment determines its connectivity and its attribute vector. Our model extends previous work on attributed stochastic block models in its ability to handle multiple continuous attributes. The continuous attributes are modeled by a Gaussian mixture model, with the assumption that the attributes for members for each community are parameterized by a unique multivariate Gaussian. Since community detection results are often difficult to validate due to the absence of a known ground truth, we quantified the ability of the fitted attributed stochastic block model to represent a particular network by performing link prediction and collaborative filtering tasks. Applying link prediction and collaborative filtering to two biological networks, we observed that the attributed SBM is useful for these applications.

Future work could extend the model to handle a combination of multiple discrete and continuous attributes. Further, while the inference or understanding of fitting a stochastic block model to weighted networks is not well understood, figuring out how to integrate edge weights and attributes in determining community structure could be useful. Next, we also mentioned the I-Louvain method, which is an extension of modularity to networks with potentially multiple, continuous attributes (Combe et al. 2015). It would be useful to characterize under what circumstances one might choose to use an SBM approach as opposed to a modularity optimization approach. We briefly discussed observed detectability properties in Fig. 3, noting that it would be interesting to characterize how the properties of the attributes and connectivity relate to effective identification of community structure. Next, whereas our specific attributed SBM model considered here does not include corrections for heterogeneous degrees, or the effect of partial dependency between attributes and connectivity, further work could broaden the diversity of networks able to be analyzed with the attributed SBM. Finally, in this current version of the attributed SBM with multiple continuous attributes, we have not developed model selection criteria to determine the number of communities, $K$, that we expect in the network. This is an important aspect of future work as it allows a user to apply the attributed SBM in a more automated manner.

Networks used across fields are becoming increasingly complex, often with multiple sources of information to integrate in order to make a conclusion for the data. Our approach to an attributed SBM advances the understanding of how to jointly consider attribute and connectivity information in a probabilistic framework.

List of Abbreviations

BMI: Body mass index; NMI: Normalized mutual information; OTU: Operational taxonomic unit; PCA: Principal component analysis; RMSE: Root mean squared error; ROC: Receiver operating characteristic; SBM: Stochastic block model 
Authors' information

Not applicable.

\section{Funding}

The research reported in this publication was supported by the NSF (ECCS-1610762). The content is solely the responsibility of the authors and does not necessarily represent the official views of the funding agency.

\section{Availability of data and materials}

Code The code to run the attributed SBM can be found here, https:/github.com/stanleyn/AttributedSBM. Data

Protein Interaction Network: The protein interaction data and its corresponding attribute information can be found in the supplementary information of this publication, https://pubs.acs.org/doi/abs/10.1021/pr401258d.

Microbiome Subject Similarity Network: The data used to compute the microbiome subject similarity network can be found here, http://datadryad.org/resource/doi:10.5061/dryad.pk75d.

\section{Competing interests}

The authors have no competing interests to declare.

\section{Author details}

${ }^{1}$ Curriculum in Bioinformatics and Computational Biology, University of North Carolina at Chapel Hill, Chapel hill, USA. 2 Present address: Stanford University, Stanford, USA. ${ }^{3}$ Department of Pharmacology \& Lineberger Comprehensive Cancer Center, University of North Carolina at Chapel Hill, Chapel hill, USA. ${ }^{4}$ Department of Computer Science, University of Salzburg, Salzburg, Austria. ${ }^{5}$ Department of Computer Science \& Biomedical Research Imaging Center, University of North Carolina at Chapel Hill, Chapel hill, USA. ${ }^{6}$ Carolina Center for Interdisciplinary Applied Mathematics, Department of Mathematics, University of North Carolina at Chapel Hill, Chapel hill, USA.

Received: 29 January 2019 Accepted: 9 July 2019

Published online: 01 August 2019

References

Aghaeepour N, Ganio EA, Mcilwain D, Tsai AS, Tingle M, Gassen SV, Gaudilliere DK, Baca Q, McNeil L, Okada R, et al (2017) An immune clock of human pregnancy. Science immunology, Vol. 2, Washington, DC

Bilmes JA, et al (1998) A gentle tutorial of the em algorithm and its application to parameter estimation for gaussian mixture and hidden markov models. Int Comput Sci Inst 4(510):126

Blondel VD, Guillaume J-L, Lambiotte R, Lefebvre E (2008) Fast unfolding of communities in large networks. J Stat Mech: Theory Exp 2008(10):P10008

Bonacci T, Audebert S, Camoin L, Baudelet E, Bidaut G, Garcia M, Witzel I-I, Perkins ND, Borg J-P, lovanna JL, et al (2014) Identification of new mechanisms of cellular response to chemotherapy by tracking changes in post-translational modifications by ubiquitin and ubiquitin-like proteins. J Proteome Res 13(5):2478-2494

Bothorel C, Cruz JD, Magnani M, Micenkova B (2015) Clustering attributed graphs: models, measures and methods. Netw Sci 3(3):408-444

Combe D, Largeron C, Géry M, Elòd E-Z (2015) I-louvain: An attributed graph clustering method. In: Advances in Intelligent Data Analysis XIV. Springer. pp 181-192

Danon L, Diaz-Guilera A, Duch J, Arenas A (2005) Comparing community structure identification. J Stat Mech: Theory Exp 2005(09):P09008

Daudin J-J, Picard F, Robin S (2008) A mixture model for random graphs. Stat Comput 18(2):173-183

Decelle A, Krzakala F, Moore C, Zdeborová L (2011) Inference and phase transitions in the detection of modules in sparse networks. Phys Rev Lett 107(6):065701

Dempster AP, Laird NM, Rubin DB (1977) Maximum likelihood from incomplete data via the em algorithm. J Royal Stat Soc Ser B (Methodol) 39(1):1-22

Deng W, Patil R, Najjar L, Shi Y, Chen Z (2014) Incorporating community detection and clustering techniques into collaborative filtering model. Proc Comput Sci 31:66-74

Fortunato S (2010) Community detection in graphs. Phys Rep 486(3-5):75-174

Fortunato S, Hric D (2016) Community detection in networks: A user guide. Phys Rep 659:1-44

Greene D, Cunningham P (2013) Producing a unified graph representation from multiple social network views. In: Proceedings of the 5th Annual ACM Web Science Conference. ACM. pp 118-121

Guinney Justin, Dienstmann Rodrigo, Wang Xin, Reyniès Ad, Schlicker A, Soneson C, Marisa L, Roepman P, Nyamundanda G, Angelino P, et al (2015) The consensus molecular subtypes of colorectal cancer. Nat Med 21(11):1350

Hric D, Peixoto TP, Fortunato S (2016) Network structure, metadata, and the prediction of missing nodes and annotations. Phys Rev X 6(3):031038

Karrer B, Newman ME (2011) Stochastic blockmodels and community structure in networks. Phys Rev E 83(1):016107

Lahti L, Salojärvi J, Salonen A, Scheffer M, Vos WMD (2014) Tipping elements in the human intestinal ecosystem. Nat Commun 5:4344

Larremore DB, Clauset A, Buckee CO (2013) A network approach to analyzing highly recombinant malaria parasite genes. PLoS Comput Biol 9(10):e1003268

Leskovec J, Lang KJ, Mahoney M (2010) Empirical comparison of algorithms for network community detection. In: Proceedings of the 19th international conference on World wide web. ACM. pp 631-640

Newman MEJ (2003) Mixing patterns in networks. Phys Rev E 67(2):026126

Newman MEJ, Clauset A (2016) Structure and inference in annotated networks. Nat Commun 7:11863

Peel L, Larremore DB, Clauset A (2017) The ground truth about metadata and community detection in networks. Sci Adv 3(5):e1602548 
Peixoto TP (2014) Efficient monte carlo and greedy heuristic for the inference of stochastic block models. Phys Rev E 89(1):012804

Porter MA, Onnela J-P, Mucha PJ (2009) Communities in networks. Not AMS 56(9):1082-1097

Shai S, Stanley N, Granell C, Taylor D, Mucha PJ (2017) Case studies in network community detection. arXiv preprint arXiv: 1705.02305

Shi Y, Larson M, Hanjalic A (2014) Collaborative filtering beyond the user-item matrix: A survey of the state of the art and future challenges. ACM Comput Surv (CSUR) 47(1):3

Snijders TAB, Nowicki K (1997) Estimation and prediction for stochastic blockmodels for graphs with latent block structure. J Classif 14(1):75-100

Soundarajan S, Hopcroft J (2012) Using community information to improve the precision of link prediction methods. In: Proceedings of the 21st International Conference on World Wide Web. ACM. pp 607-608

Taylor D, Shai S, Stanley N, Mucha PJ (2016) Enhanced detectability of community structure in multilayer networks through layer aggregation. Phys Rev lett 116(22):228301

Wang P, BaoWen X, YuRong W, Zhou X (2014) Link prediction in social networks: the state-of-the-art. arXiv preprint arXiv:1411.5118

Yang J, Leskovec J (2015) Defining and evaluating network communities based on ground-truth. Knowl Inf Syst 42(1):181-213

Yang J, McAuley J, Leskovec J (2013) Community detection in networks with node attributes. In: Data mining (ICDM), 2013 ieee 13th international conference on. IEEE. pp 1151-1156

Zhang P, Krzakala F, Reichardt J, Zdeborová L (2012) Comparative study for inference of hidden classes in stochastic block models. J Stat Mech: Theory Exp 2012(12):P12021

\section{Publisher's Note}

Springer Nature remains neutral with regard to jurisdictional claims in published maps and institutional affiliations.

\section{Submit your manuscript to a SpringerOpen ${ }^{\odot}$} journal and benefit from:

- Convenient online submission

- Rigorous peer review

Open access: articles freely available online

- High visibility within the field

- Retaining the copyright to your article

Submit your next manuscript at $\gg$ springeropen.com 